\title{
Simultaneous ultrafast probing of intramolecular vibrations and photoinduced charge carriers in rubrene using broadband time-domain $\mathrm{THz}$ spectroscopy
}

\author{
Mattijs Koeberg, ${ }^{1,2}$ Euan Hendry, ${ }^{3}$ Juleon M. Schins, ${ }^{4}$ Hendrik A. van Laarhoven, ${ }^{5}$ Cees F. J. Flipse, ${ }^{5}$ Klaus Reimann, ${ }^{6}$ \\ Michael Woerner, ${ }^{6}$ Thomas Elsaesser, ${ }^{6}$ and Mischa Bonn ${ }^{1,2}$ \\ ${ }^{1}$ FOM Institute for Atomic and Molecular Physics, Kruislaan 407, 1098 SJ Amsterdam, The Netherlands \\ ${ }^{2}$ Leiden Institute of Chemistry, Leiden University, Einsteinweg 55, P.O. Box 9502, 2300 RA Leiden, The Netherlands \\ ${ }^{3}$ Electromagnetic Materials Group, School of Physics, University of Exeter, Stocker Road, Exeter EX4 4QL, United Kingdom \\ ${ }^{4}$ Opto-Electronic Materials Section, DelftChemTech, Delft University of Technology, Mekelweg 15, 2629 JB Delft, The Netherlands \\ ${ }^{5}$ Chemical Engineering and Chemistry, Eindhoven University of Technology, $5600 \mathrm{MB}$ Eindhoven, The Netherlands \\ ${ }^{6}$ Max-Born-Institut für Nichtlineare Optik und Kurzzeitspektroskopie, Max-Born-Strasse 2A, 12489 Berlin, Germany
}

(Received 22 January 2007; revised manuscript received 15 March 2007; published 29 May 2007)

\begin{abstract}
We determine the ultrafast frequency- and time-resolved complex dielectric responses of photoexcited, single-crystal rubrene in the frequency range of $10-30 \mathrm{THz}\left(330-1000 \mathrm{~cm}^{-1}\right)$ using ultrafast broadband $\mathrm{THz}$ spectroscopy. In this frequency range, we observe the response of both photogenerated mobile charges and intramolecular vibrational modes simultaneously, both of which vary with time after excitation. The data in conjunction with a theoretical model indicate a dynamic blueshift of the $15.5 \mathrm{THz}$ phonon.
\end{abstract}

DOI: 10.1103/PhysRevB.75.195216

PACS number(s): 72.80.Le, 63.20.Kr, 72.20.-i, 71.38.-k

In most semiconductors, there is strong coupling between longitudinal optical (LO) phonons and mobile electrons ${ }^{1}$ and plasmons. ${ }^{2}$ These interactions generally greatly affect the charge mobility in these materials. With its high sensitivity to charge mobility, terahertz time-domain spectroscopy (THzTDS) has proven a valuable technique for studying electronphonon coupling in different materials, including titanium dioxide, ${ }^{3}$ sapphire, ${ }^{4}$ and organic semiconductors. ${ }^{5-7}$ In these reports, information on the nature of charge transport and electron-phonon interactions has generally been inferred indirectly from temperature-dependent measurements at relatively low $\mathrm{THz}$ frequencies $(<3 \mathrm{THz})$, where the response is dominated by the dynamics and mobility of the photocharge carriers.

With the development of ultrafast lasers and advanced detection schemes, broadband THz-TDS (BBTHz-TDS) with a $\mathrm{THz}$ frequency range typically from 10 to $30 \mathrm{THz}-$ has recently become accessible ${ }^{8-11}$ and has been proven extremely fruitful in elucidating charge carrier dynamics in, for instance, semiconductors, ${ }^{2}$ carbon nanotubes, ${ }^{12}$ and semimetals. ${ }^{13}$ In addition to the improved time resolution enabling an increased bandwidth of the $\mathrm{THz}$ radiation, the broadband scheme in principle allows for the simultaneous detection of charge and phonon response, as phonon modes typically lie within this frequency range.

Indeed, BBTHz-TDS has recently been applied to study the dynamics of LO phonon-plasmon coupled excitations in indium phosphide, ${ }^{2}$ where the authors found that the coupled phonon-plasmon resonances were not established instantaneously on photoexcitation. Instead, both the phonon and plasmon frequencies shifted with time after excitation, eventually forming a mixed mode within one oscillation cycle of the phonon, typically on subpicosecond time scales, in this inorganic semiconductor.

Here, we apply broadband $\mathrm{THz}$ spectroscopy to study carrier-phonon interactions in organic semiconductors (i.e., conjugated molecular crystals ${ }^{14}$ ). For these materials, vibrational (phonon) modes may be separated into those that are delocalized over more than one molecular unit (termed intermolecular vibrations) and localized intramolecular vibrations. Several studies, both theoretical ${ }^{15-18}$ and experimental, ${ }^{19-22}$ have suggested that the coupling of electrons to both intermolecular and intramolecular vibrations should play an important role in the charge transport properties, especially for the acenes. ${ }^{14}$ For pentacene, an electronphonon spectrum in the $1-8 \mathrm{THz}$ range (corresponding to intermolecular vibrations), determined using a quantum tunneling experiment, suggested that these modes did indeed couple to free charges, ${ }^{19}$ although the coupling was erroneously associated with superconductivity. In any case, no direct time-resolved spectroscopic evidence of coupling between (photoinduced) mobile charges and intramolecular vibrations (which lie in the $10-30 \mathrm{THz}$ frequency range) exists for this class of materials.

We present optical pump-broadband $\mathrm{THz}$ probe spectroscopy on single crystal rubrene, allowing simultaneous access to the electronic and intramolecular vibrational responses in the desired 10-30 THz frequency range. As the electric field of the transmitted $\mathrm{THz}$ probe pulse is detected directly in the time domain, both amplitude and phase information are obtained, providing access to the photoinduced complex dielectric response of the sample. Owing to the ultrashort detection pulses, the dynamics of the frequency-resolved spectral changes can be followed on femtosecond time scales.

We use $30 \mathrm{fs}, 400 \mathrm{~nm}(3.0 \mathrm{eV})$ pump pulses to induce an electronic transition in rubrene $\left(S_{0}-S_{1}\right.$ gap $\left.1.92-2.2 \mathrm{eV}\right){ }^{23}$ resulting in intramolecular excitations and generating mobile charges, as has been shown previously for acenes. ${ }^{5-7}$ The crystals were vapor grown using several vacuum sublimation steps, and as a result, achieving trap densities in the order of $10^{15} \mathrm{~cm}^{-3} \cdot{ }^{24}$ Recent work has demonstrated the propensity of rubrene to oxidize ${ }^{25}$ and the effect of oxidation on the band structure ${ }^{26}$ and photoconductivity. ${ }^{27}$ Although most of the experiments shown here were performed in air, we found no indications for effects due to oxidation in our experiment. No change in the sample response was observable in the course 

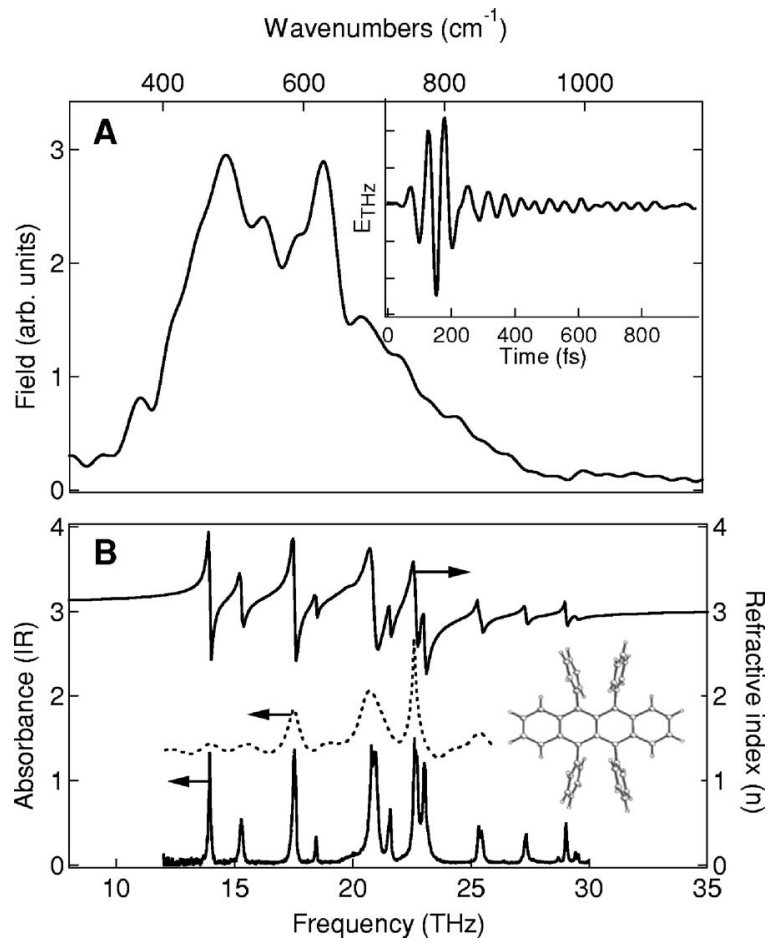

FIG. 1. (a) THz probe pulse in the frequency and time domain (inset). (b) Linear IR absorption spectrum of the rubrene crystal measured with an IR spectrometer (solid line) and extracted from the TD-THz experiment (dotted line, offset vertically, see text). Note the saturation of some of the peaks in the IR spectrum-the actual absorbance may be higher for some of the peaks. The molecular structure (Ref. 28) of rubrene is shown as an inset in (b).

of time and, more importantly, measurements on fresh samples under vacuum revealed identical dynamics at 1-3 THz frequencies as those reported here.

The thickness of the rubrene crystals was several micrometers, well exceeding the optical penetration depth at $400 \mathrm{~nm}(\sim 1 \mu \mathrm{m})$, ensuring all excitation photons $(\sim 2$ $\times 10^{21} / \mathrm{m}^{2}$ per pulse) were absorbed. We use the THz probe pulses shown in Fig. 1(a) (full width at half maximum $<200 \mathrm{fs}$ ) to probe the dielectric response in the frequency range of $10-30 \mathrm{THz}\left(330-1000 \mathrm{~cm}^{-1}\right)$ as a function of delay time between pump and probe pulses. The THz transients were recorded at constant delay time between the pump pulse and the $\mathrm{THz}$ sampling pulse. The probe transmission was measured through the $a-b$ plane of the crystal ${ }^{28}$ Further experimental details have been described previously. ${ }^{10}$

In the frequency range of the $\mathrm{THz}$ probe, rubrene shows several IR active vibrational modes. Figure 1(b) depicts the ground-state IR absorption of an $\sim 5$ - $\mu$ m-thick rubrene crystal. The frequency resolution of the THz-TDS experiment is illustrated in the same figure showing an absorption spectrum obtained by using $A_{T H z}=-\log \left[E_{s}(\omega) / E_{r}(\omega)\right]$, in which $E_{s}(\omega)$ and $E_{r}(\omega)$ are the Fourier transforms (amplitude) of the time-domain $\mathrm{THz}$ electric fields transmitted through the sample and through air, respectively. The observed vibrational transitions are due to intramolecular vibrations, while intermolecular vibrations are limited to lower frequencies $(<6 \mathrm{THz}) \cdot{ }^{19,22,29}$

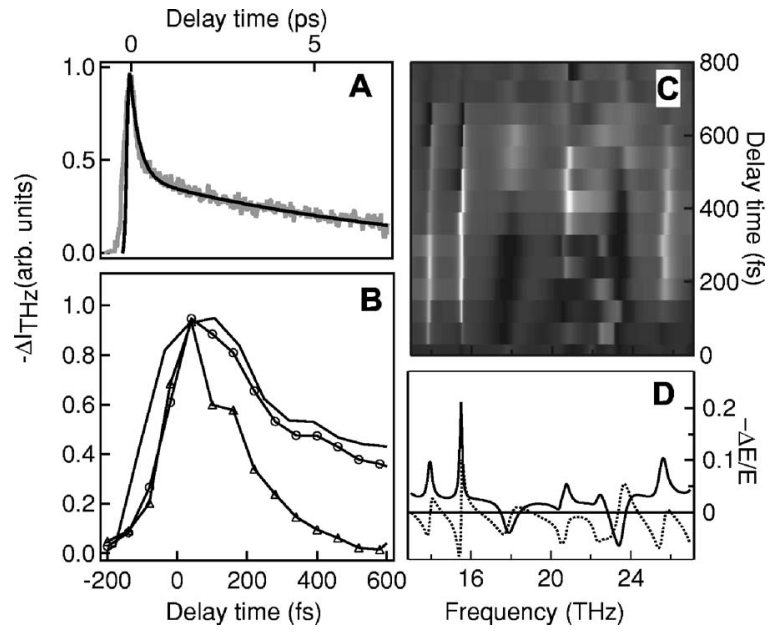

FIG. 2. (a) Photoinduced changes in THz transmission through rubrene as a function of delay time after photoexcitation, measured with a $\mathrm{HgCdTe}$ detector (grey line). Black solid line is the result of the calculation described in the text. (b) Dynamics on short time scales. The line marks the $\mathrm{HgCdTe}$ detector result; the marked traces represent dynamics inferred from time- and frequencyresolved experiments: the signal integrated over all frequencies (circles) and the purely resonant signal (resonant minus nonresonant) at $15.5 \mathrm{THz}$ (integrated from 15 to $16 \mathrm{THz}$, triangles). (c) First $800 \mathrm{fs}$ of the real part of the differential signal $\Delta E / E$; the horizontal frequency axis corresponds with that of the bottom figure. Linear gray scale from 0.2 (white) to -0.05 (black). (d) The real (full line) and imaginary (dotted line) parts of $\Delta E / E$ at the maximum of the signal (integrated from 200 to $400 \mathrm{fs}$ ).

To determine the dynamics of the photoinduced $\mathrm{THz}$ absorption, the change in transmitted $\mathrm{THz}$ intensity (induced absorption) $\Delta I(\tau)$ is measured with a time-integrating $\mathrm{HgCdTe}$ detector as a function of delay time $\tau$ after the $400 \mathrm{~nm}$ excitation pulse. The $\Delta I(\tau)$ trace for rubrene [Fig. $2(\mathrm{a})]$ shows fast (described by an exponential time constant of $\sim 0.2 \mathrm{ps})$ and slow $(\sim 6 \mathrm{ps})$ components. We observe a similar bimodal behavior when probing in the low-frequency range $(\sim 1 \mathrm{THz})$, in agreement with previous reports ${ }^{5-7}$ indicating that these dynamics are dominated by charge carrier response. The single-crystal nature of our sample is reflected in the anisotropy of the $\Delta I$ signal: $\Delta I$ is a factor of 5 larger if the $b$ axis of the crystal (the directions with strongest $\pi$-electron overlap between neighboring molecules) is parallel to the $\mathrm{THz}$ probe beam polarization, rather than the $a$ axis. This is in agreement with both theoretically predicted ${ }^{30}$ and measured field effect mobility ${ }^{28,31}$ anisotropy of acene single crystals.

A time-dependent frequency spectrum of the transmitted THz field $\Delta E(\omega, \tau)$ is obtained from a Fourier transform of the difference between the time domain $\mathrm{THz}$ pulses before and after photoexcitation for varying pump-probe delays [Fig. 2(b)]. As shown in Figs. 2(c) and 2(d), the data consist of distinct resonances in the first $700 \mathrm{fs}$, superimposed on a broad absorption, which extends over the full frequency range. The bimodal time evolution of the photoinduced signal at the nonresonant frequency of $16.3 \mathrm{THz}$ simply follows the frequency integrated dynamics [i.e., $\Delta I(\tau)]$, and is iden- 
tical to the response observed at low frequencies, which has been shown to be related to carrier mobility ${ }^{5-7}$ and may be characterized by a Drude-type response. In contrast, the resonant signal at $15.5 \mathrm{THz}$ decays much faster [Fig. 2(b)]. This difference suggests that the nature of the excitation associated with the fast and slow components is different. Both are presumably associated with charge redistribution. ${ }^{5-7}$ The fast response is induced directly by photoexcitation of rubrene chromophores. Such vibronic excitation is connected with a change of the intramolecular electron distribution, affecting the vibrational potentials and frequencies. We attribute the fast spectral shifts to this effect, decaying with the depopulation of the initially excited state. Here, both intramolecular relaxation (internal conversion) and charge ejection into the environment may contribute. As is obvious from the data, after $2 \mathrm{ps}$ there still exist mobile charges, but there is no significant effect on the vibrational frequency anymore.

However, interference of the narrow ground-state resonances with the carrier-induced broadband (Drude-type) response will necessarily cause signals in the transient spectrum at the frequencies corresponding to the ground-state resonances. ${ }^{32}$ Indeed, the transient $\mathrm{THz}$ spectra [Fig. 2(c)] exhibit features corresponding to the ground-state absorption spectrum [Fig. 1(b)]. Information about interactions between the electrons (or excited states) and phonons therefore has to be retrieved from the data by a model that takes into account this effect. Our analytical model of $\mathrm{THz}$ propagation in a photoexcited sample relates the normalized pump-induced change in transmission $\Delta E(\omega, \tau)$ to the time-dependent sample properties, neglecting dipole-dipole interactions, but including the Fresnel reflections off the front sample surface, ${ }^{33}$

$$
\frac{\Delta E_{I}\left(L, \omega, \omega_{p}\right)}{E_{t r}(L, \omega)}=C\left(\omega, \omega_{p}\right)\left[A_{\text {Drude }}\left(\omega, \omega_{p}\right)+A_{e x c}\left(\omega, \omega_{p}\right)\right],
$$

where $\omega_{p}$ is the frequency variable corresponding to the pump delay time $\tau$. The factor $C$ contains the ground-state properties of rubrene and describes the propagation of the $\mathrm{THz}$ pulse and reflection from the different interfaces. The two terms $A$ account for the excitation process: $A_{\text {Drude }}$ contains a broad, featureless (Drude) excited-state response characterized by $\chi(\omega)=1 /[-i \omega(2 \gamma-i \omega)]$, with a scattering rate $\gamma . A_{e x c}$ is a term that describes a transient shift of the phonon response, presumably caused by electron-phonon interactions. This transient shift is characterized by a magnitude of the shift of the resonance, and an exponential time that defines its return to the original frequency.

This treatment, analogous to that presented in Ref. 34, will be detailed in a separate publication. ${ }^{33}$ In brief, the treatment takes into account the spatial variations of the pump intensity along the direction of excitation (determined by the optical absorption coefficient), ground-state sample dispersion with multiple resonant features, dispersion between the group velocity of the pump, and the $\mathrm{THz}$ phase velocity. The detector response function is also accounted for. ${ }^{33}$ The parameters describing the ground-state absorption are taken from a fit to the Fourier transform infrared (FTIR) spectrum
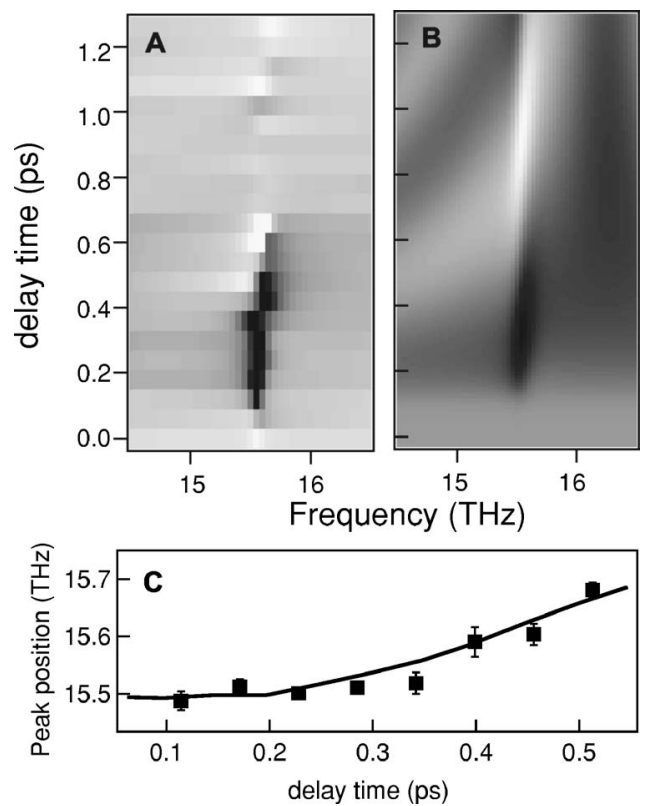

FIG. 3. Comparison of the experimental $\Delta E / E$ data around $15.5 \mathrm{THz}$ (a) with the modeled data at the same frequency range (b). Scale as in Fig. 2, but inverted for clarity, (see text for details). Panel (c) depicts the central frequency of both the experimental (squares) and modeled (solid line) data obtained from Lorentzian fits to horizontal cuts through panels (a) and (b).

shown in Fig. 1(b), while the Drude scattering rate (which hardly influences the results) is taken as $105 \times 10^{12} \mathrm{rad} \mathrm{s}^{-1}$ following Ref. 31. Optimum agreement with the measured data was obtained when the widths of the ground-state absorption resonances are slightly increased with respect to the fit parameters determined from the FTIR spectrum. Additional input parameters that can be determined independently are the sample thickness, the pump penetration depth, the free electron lifetime for the Drude term $A_{\text {Drude, }}$, and the exponential recovery time for the excitation-induced phonon frequency shift amounting to $\sim 6$ and $200 \mathrm{fs}$, respectively. The two lifetimes can be determined from the data in Fig. 2(a).

Two key features of the data can only be reproduced by the model when electron-phonon interactions are taken into account, incorporated in the term $A_{e x c}$, which represents the carrier-induced phonon shift: First, the dynamics of the vibrational features (measured at the peaks in the spectrum) and nonresonant response are very different: At delay times $\tau>700 \mathrm{fs}$, when the frequency-integrated signal has decreased to $\sim 50 \%$ (see Fig. 2), the resonant features have decreased much more than the broad, nonresonant response. Second, the $\Delta E(\omega, \tau) / E(\omega)$ data reveal shifts in frequency: the response is not aligned vertically in Fig. 2(c) - this is more apparent in the close-up depicted in Fig. 3(a) and the derived peak position depicted in Fig. 3(c). These figures show the response of the $15.5 \mathrm{THz}$ mode, which we have analyzed in more detail. The data in Fig. 3(a) can be reproduced [Fig. 3(b)] only if an induced shift of the THz resonance frequency is incorporated into the model. For the $15.5 \mathrm{THz}$ mode, a dynamic blueshift of $0.8 \pm 0.4 \mathrm{THz}$ is required to reproduce the data. The sign of the shift is apparent 
from the obliqueness of the resonance line: both the positive and negative extrema of the measured resonance at $15.5 \mathrm{THz}$ [see Fig. 3(b)] shift to higher frequencies as a function of pump delay time. This is emphasized in Fig. 3(c), which depicts the peak of the resonance obtained from Lorentzian fits to both the experimental and modeled data. Note that the actually observed shift $(\sim 0.2 \mathrm{THz})$ is smaller than the $0.8 \mathrm{THz}$ shift obtained from the model. This can be understood by noting that the response observed in the timefrequency representation [Fig. 2(c), recorded here according to scheme I in Ref. 34] is not only determined by the magnitude of the shift, but also by the dynamics of that shift. The $200 \mathrm{fs}$ lifetime of the shift is very short and thus affects the observed spectral dynamics significantly. In the limit of infinite lifetime, the true phonon shift would be recovered.

If the shift of the phonon mode is not incorporated in the model, both the dynamics and spectral response (the relatively fast resonant response and the dispersion clearly observed in the experimental data) cannot be adequately reproduced. For the other experimental modes, the shift is smaller and/or a larger error is associated with the magnitude of the shift due to larger ground-state absorption. The same calculation also nicely reproduces the spectrally integrated response of Fig. 2(a) (black line in upper panel). The magnitude of the excitation-induced shift of the phonon resonance provides a direct measure of the electron-phonon interaction strength. As such, the technique presented here in conjunction with the theoretical analysis allows one to quantify electron-phonon interactions. It should be noted that we cannot say with certainty whether the observed vibrational re- sponse originates from interactions with bound electron-hole states (exciton), free electrons, or free holes.

Indications for a large dynamic coupling between the 15.5 THz mode and charge carriers have been obtained previously from a differential IR absorption measurement, which, in addition to a spectrally broad Drude-type carrier response for the free electrons, reveals changes in the $\sim 15 \mathrm{THz}$ region. ${ }^{31,32}$ The approach presented here allows for a quantification of the electron-phonon coupling strength through the magnitude of the induced dynamic shift.

To summarize, we have reported the photoinduced femtosecond time-resolved dielectric response of a rubrene crystal in the frequency range of $10-30 \mathrm{THz}$. On top of a broad response from the photoinduced mobile charges, spectral features are observed at ground-state resonances. The dynamic behavior of these charge-induced features contains information on electron-phonon interactions. This demonstrates that broadband time-domain $\mathrm{THz}$ spectroscopy is a promising technique to study the photoinduced electronic and vibronic responses, as well as their cross terms (electron-phonon coupling) in organic crystals.

The authors would like to thank A. Morpurgo for supplying the samples and J. van den Brink, H. G. Muller, and J. Dreyer for helpful discussions. This work is part of the research program of the "Stichting voor Fundamenteel Onderzoek der Materie (FOM)," which is financially supported by the "Nederlandse Organisatie voor Wetenschappelijk Onderzoek (NWO)." The measurements at the Max-Born-Institut were supported by the Large Scale Laser Facilities program.
${ }^{1}$ D. Emin, Phys. Rev. B 48, 13691 (1993).

${ }^{2}$ R. Huber, C. Kübler, S. Tübel, A. Leitenstorfer, Q. T. Vu, H. Haug, F. Kohler, and M. C. Amann, Phys. Rev. Lett. 94, 027401 (2005).

${ }^{3}$ E. Hendry, F. Wang, J. Shan, T. F. Heinz, and M. Bonn, Phys. Rev. B 69, 081101(R) (2004).

${ }^{4}$ J. Shan, F. Wang, E. Knoesel, M. Bonn, and T. F. Heinz, Phys. Rev. Lett. 90, 247401 (2003).

${ }^{5}$ F. A. Hegmann, R. R. Tykwinski, K. P. H. Lui, J. E. Bullock, and J. E. Anthony, Phys. Rev. Lett. 89, 227403 (2002).

${ }^{6}$ V. K. Thorsmolle, R. D. Averitt, X. Chi, D. J. Hilton, D. L. Smith, A. P. Ramirez, and A. J. Taylor, Appl. Phys. Lett. 84, 891 (2004).

${ }^{7}$ O. Ostroverkhova, D. G. Cooke, F. A. Hegmann, J. E. Anthony, V. Podzorov, M. E. Gershenson, O. D. Jurchescu, and T. T. M. Palstra, Appl. Phys. Lett. 88, 162101 (2006).

${ }^{8}$ R. Huber, A. Brodschelm, F. Tauser, and A. Leitenstorfer, Appl. Phys. Lett. 76, 3191 (2000).

${ }^{9}$ K. Reimann, R. P. Smith, A. M. Weiner, T. Elsaesser, and M. Woerner, Opt. Lett. 28, 471 (2003).

${ }^{10}$ C. Luo, K. R. M. Woerner, and T. Elsaesser, Appl. Phys. A: Mater. Sci. Process. 78, 435 (2004).

${ }^{11}$ C. Kubler, R. Huber, and A. Leitenstorfer, Semicond. Sci. Technol. 20, S128 (2005).

${ }^{12}$ L. Perfetti, T. Kampfrath, F. Schapper, A. Hagen, T. Hertel, C.
Aguirre, P. Desjardins, R. Martel, C. Frischkorn, and M. Wolf, Phys. Rev. Lett. 96, 027401 (2006).

${ }^{13}$ Tobias Kampfrath, Luca Perfetti, Florian Schapper, Christian Frischkorn, and Martin Wolf, Phys. Rev. Lett. 95, 187403 (2005).

${ }^{14}$ N. Karl, Synth. Met. 133, 649 (2003).

${ }^{15} \mathrm{~K}$. Hannewald and P. A. Bobbert, Phys. Rev. B 69, 075212 (2004).

${ }^{16}$ T. Kato and T. Yamabe, J. Chem. Phys. 120, 3311 (2004).

${ }^{17}$ L. Giuggioli, J. D. Andersen, and V. M. Kenkre, Phys. Rev. B 67, 045110 (2003).

${ }^{18}$ A. Devos and M. Lannoo, Phys. Rev. B 58, 8236 (1998).

${ }^{19}$ M. Lee, J. H. Schon, C. Kloc, and B. Batlogg, Phys. Rev. Lett. 86, 862 (2001).

${ }^{20}$ V. Coropceanu, M. Malagoli, D. A. da Silva Filho, N. E. Gruhn, T. G. Bill, and J. L. Bredas, Phys. Rev. Lett. 89, 275503 (2002).

${ }^{21}$ N. E. Gruhn, D. A. d. Silva, T. G. Bill, M. Malagoli, V. Coropceanu, A. Kahn, and J. L. Bredas, J. Am. Chem. Soc. 124, 7918 (2002).

${ }^{22}$ T. Hasche, T. W. Canzler, R. Scholz, M. Hoffmann, K. Schmidt, T. Frauenheim, and K. Leo, Phys. Rev. Lett. 86, 4060 (2001).

${ }^{23}$ Z. Y. Xie, J. Feng, J. S. Huang, S. Y. Liu, Y. Wang, and J. C. Shen, Synth. Met. 108, 81 (2000).

${ }^{24}$ R. W. I. de Boer, M. E. Gershenson, A. F. Morpurgo, and V. Podzorov, Phys. Status Solidi 201, 1302 (2004). 
${ }^{25}$ D. Kafer and G. Witte, Phys. Chem. Chem. Phys. 7, 2850 (2005).

${ }^{26}$ Oleg Mitrofanov, David V. Lang, Christian Kloc, J. Magnus Wikberg, Theo Siegrist, Woo-Young So, M. A. Sergent, and Arthur P. Ramirez, Phys. Rev. Lett. 97, 166601 (2006).

${ }^{27}$ Hikmat Najafov, Ivan Biaggio, Vitaly Podzorov, Matthew F. Calhoun, and Michael E. Gershenson, Phys. Rev. Lett. 96, 056604 (2006).

${ }^{28}$ V. C. Sundar, J. Zaumseil, V. Podzorov, E. Menard, R. L. Willett, T. Someya, M. E. Gershenson, and J. A. Rogers, Science 303, 1644 (2004).

${ }^{29}$ D. A. Tenne, S. Park, T. U. Kampen, A. Das, R. Scholz, and D. R. T. Zahn, Phys. Rev. B 61, 14564 (2000).

${ }^{30}$ G. A. d. Wijs, C. C. Mattheus, R. A. de Groot et al., Synth. Met. 139, 109 (2003).

${ }^{31}$ V. Podzorov, E. Menard, A. Borissov, V. Kiryukhin, J. A. Rogers, and M. E. Gershenson, Phys. Rev. Lett. 93, 086602 (2004).

${ }^{32}$ For a thin excitation region in the sample, and for small modulations of the $\mathrm{THz}$ field [i.e., $\Delta E(\omega, \tau) \ll E(\omega)$ ] the ratio $\Delta E(\omega, \tau) / E(\omega)$ is directly proportional to the photoinduced changes in the complex refractive index $\Delta n$ [see, e.g., E. Knoesel, M. Bonn, J. Shan, F. Wang, and T. F. Heinz, J. Chem. Phys. 121, 394 (2004)]. $\Delta n$ in turn can be related to the change in the dielectric function $\Delta \varepsilon$ by considering $\left(n_{0}+\Delta n\right)^{2}=\varepsilon_{0}+\Delta \varepsilon$, where $n_{0}$ and $\varepsilon_{0}$ are the ground-state refractive index and dielectric function. For small changes in refractive index $\left(\Delta n \ll n_{0}\right), \Delta n$ $\approx \Delta \varepsilon / 2 n_{0}$, so that pronounced absorptions in the ground state will also appear in the $\Delta E(\omega, \tau) / E(\omega)$ spectrum.

${ }^{33}$ J. Schins, E. Hendry, M. Bonn, and H. G. Muller (unpublished).

${ }^{34}$ H. Nemec, F. Kadlec, and P. Kuzel, J. Chem. Phys. 117, 8454 (2002). 